PROCEEDINGS OF THE

AMERICAN MATHEMATICAL SOCIETY

Volume 91, Number 1, May 1984

\title{
MOST MAPS OF THE PSEUDO-ARC ARE HOMEOMORPHISMS
}

\author{
WAYNE LEWIS
}

\begin{abstract}
We prove the following results. (1) If $M(P)$ is the space of maps of the pseudo-arc into itself with the sup metric, then the subset $\hat{H}(P)$ of maps of the pseudo-arc into itself which are homeomorphisms onto their images is a dense $G_{\delta}$ in $M(P)$. (2) Every homeomorphism of the pseudo-arc onto itself is a product of $\epsilon$-homeomorphisms. (3) There exists a nonidentity homeomorphism of the pseudo-arc with an infinite sequence of $p$ th roots. (4) Every map between chainable continua can be lifted to a homeomorphism of pseudo-arcs.
\end{abstract}

We will investigate certain properties of maps and homeomorphisms of the pseudo-arc and other chainable continua. The pseudo-arc is characterized [2] as a nondegenerate, hereditarily indecomposable, chainable continuum. In most cases we will be defining maps or investigating their behavior in terms of functions between defining sequences of chains and patterns. For definitions of chain, link, pattern, amalgamation, and crooked, and for basic properties of these, the reader is referred to $[\mathbf{1}, \mathbf{2}, \mathbf{9}$, and 12]. Unless otherwise specified all maps are surjections.

Some specific facts we will make use of are the following.

LEMMA 1 [2]. If $X$ is a nondegenerate chainable continuum such that for each chain $C$ covering $X$ and $\epsilon>0$ there is a chain $D$ of mesh less than $\epsilon$ which covers $X$ and is crooked in $C$, then $X$ is a pseudo-arc.

Lemma 2 [1]. If $P$ is a pseudo-arc, $C$ is a chain covering $P$, and $f$ is a pattern onto $C$, then there is a chain $D$ covering $P$ which closure refines the chain $C$ and follows the pattern $f$ in $C$.

The chain $D$ can be obtained by amalgamation of any chain of sufficiently small mesh in a defining sequence of chains for $P$.

LEMMA $3[\mathbf{1}] . \quad$ Let $\left\{C_{i}\right\}_{i=1}^{\infty}$ and $\left\{D_{i}\right\}_{i=1}^{\infty}$ be two sequences of chains covering the chainable continuum $X$ such that (1) for each $i, C_{i}$ and $D_{i}$ have the same number of links, (2) for some $N$ and each $i>N, \operatorname{mesh}\left(C_{i}\right)<1 / i$ and $\operatorname{mesh}\left(D_{i}\right)<1 / i$, and (3) for each $i$, there exists a pattern $f_{i}$ such that $C_{i+1}$ follows $f_{i}$ in $C_{i}$ and $D_{i+1}$ follows $f_{i}$ in $D_{i}$.

Received by the editors September 21, 1982 and, in revised form, August 3, 1983. The results of this paper were included as part of a talk given to the meeting of the American Mathematical Society in Baton Rouge, Louisiana, November 1982.

1980 Mathematics Subject Classification. Primary 54F20; Secondary 54H15.

Key words and phrases. Pseudo-arc, chainable continuum, $\epsilon$-homeomorphism, space of homeomorphisms. 
Then there exists a homeomorphism $h: X \rightarrow X$ such that $h\left(C_{i}(j)\right) \subset \operatorname{st}\left(D_{i}(j), D_{i}\right)^{*}$ for each link $C_{i}(j)$ of $C_{i}$.

Every homeomorphism of a chainable continuum onto itself is determined by, and determines, sequences of chains as in Lemma 3. Every map between chainable continua is determined by, and determines, such sequences of chains with the modification that no mesh $\left(C_{i}\right)$ need be small and the $D_{i}$ 's are chains covering the image.

Since one lemma in our discussion of the space of maps of the pseudo-arc will be used in another section, we look at that first.

1. Homeomorphisms in $M(P)$. Let $M(P)$ be the space of maps of the pseudo-arc $P$ into itself with the sup metric. Let $\hat{H}(P)$ be the subspace of $M(P)$ consisting of all maps of the pseudo-arc, $h: P \rightarrow P$, with $h$ a homeomorphism of $P$ onto $h(P)$ (not necessarily all of $P$ ). We will show that $\hat{H}(P)$ is a dense $G_{\delta}$ in $M(P)$. We will break the argument up into a few smaller steps.

Lemma 1.1. Let $P$ be a pseudo-arc, $\hat{P}$ be a nondegenerate subcontinuum of $P$, and $\left\{C_{i}\right\}_{i=1}^{n}$ and $\left\{D_{i}\right\}_{i=1}^{n}$ be finite sequences of chains covering $P$ and $\hat{P}$ respectively such that (1) for each $i, C_{i}$ and $D_{i}$ have the same number of links, and (2) for each $i<n$, there exists a pattern $f_{i}$ which $C_{i+1}$ follows in $C_{i}$ and $D_{i+1}$ follows in $D_{i}$.

Then there exists a homeomorphism $h: P \rightarrow \hat{P}$ such that $h\left(C_{i}(j)\right) \subset \operatorname{st}\left(D_{i}(j), D_{i}\right)^{*}$ for each link $C_{i}(j)$ of $C_{i}$.

Proof. By repeated applications of Lemmas 1 and 2 of the introduction, we can continue to construct sequences of chains $\left\{C_{i}\right\}_{i=n+1}^{\infty}$ and $\left\{D_{i}\right\}_{i=n+1}^{\infty}$ such that $\left\{C_{i}\right\}_{i=1}^{\infty}$ and $\left\{D_{i}\right\}_{i=1}^{\infty}$ satisfy the hypotheses of Lemma 3 of the introduction.

Lemma 1.2. $\hat{H}(P)$ is dense in $M(P)$.

Proof. Let $f \in M(P)$ and $\epsilon>0$. If $f(P)$ is a constant, by the hereditary equivalence of $P[\mathbf{1 2}]$ there is a subcontinuum $\hat{P}$ of $P$ within the $\epsilon$-neighborhood of $f(P)$ and a homeomorphism $h: P \rightarrow \hat{P}$. Then $\operatorname{dist}(f, h)<\epsilon$.

If $f(P)$ is nondegenerate, let $D_{1}$ be a chain of mesh less than $\epsilon / 2$ covering $f(P)$, and let $C_{1}$ be the chain $f^{-1}\left(D_{1}\right)$ covering $P$. By Lemma 1.1 there is a homeomorphism $h: P \rightarrow f(P)$ such that $h\left(C_{1}(j)\right) \subset \operatorname{st}\left(D_{1}(j), D_{1}\right)^{*}$ for each link $C_{1}(j)$ of $C_{1}$. This means that $\operatorname{dist}(f, h)<\epsilon$.

Lemma 1.3. $\hat{H}(P)$ is a $G_{\delta}$ in $M(P)$.

Proof. For each positive integer $i$, let $M_{i}(P)$ be the maps of $P$ into itself with each point inverse having diameter less than $1 / i$. Then $M_{i}(P)$ is open, and $\hat{H}(P)=$ $\bigcap_{i=1}^{\infty} M_{i}(P)$.

Theorem 1.4. $\hat{H}(P)$ is a dense $G_{\delta}$ in $M(P)$.

Proof. Lemmas 1.2 and 1.3 .

In [2] R. H. Bing showed that for any Euclidean space or Hilbert space $E$ of dimension greater than 1 the collection of subcontinua of $E$ which are pseudo-arcs is a dense $G_{\delta}$ in the hyperspace of subcontinua of $E$. Thus, in at least one sense, most continua are pseudo-arcs, and most maps of pseudo-arcs are homeomorphisms.

It then seems natural to ask whether most maps between continua are homeomorphisms of pseudo-arcs. If $X$ is a metric space, the collection $M C(X)$ of maps 
between subcontinua of $X$ can be topologized in a natural way by defining the distance between two elements $f$ and $g$ of $M C(X)$ (possibly with different domains) to be the Hausdorff distance between their graphs in $X \times X$.

However, even though all continua can be approximated by pseduo-arcs, and all maps of pseudo-arcs can be approximated by homeomorphisms of pseudo-arcs, in dimension 2 at least there are maps between subcontinua which cannot be approximated by maps (or homeomorphisms) of pseudo-arcs. For example, let $A$ be the arc $[-\pi / 4,3 \pi / 4]$ on the $x$-axis in the plane, and $f: A \rightarrow E^{2}$ be given by $f(\theta)=$ $(r, \theta)$ where $r=\sin 4 \theta$ in polar coordinates. While both the domain and image of this map can be approximated by pseudo-arcs in the plane, the map cannot be approximated by maps between pseudo-arcs in the plane.

The case is different in dimension 3 or greater. There one can use general position, and combine the arguments of Bing and the author to obtain the following result.

THEOREM 1.5. If $E$ is a Euclidean space or Hilbert space of dimension 3 or greater, then the collection of homeomorphisms between pseudo-arcs is a dense $G_{\delta}$ in $M C(E)$.

Note that in Theorem 1.5, even if the element $f$ of $M C(E)$ has the same domain and range, an approximating homeomorphism of pseudo-arcs will in general have domain and range which are distinct pseudo-arcs.

2. $\epsilon$-homeomorphisms. There has been some interest in the structure of the space of homeomorphisms of the pseudo-arc, though at present very little is known about it. Brechner [5] has asked whether it is connected, has nondegenerate connected subsets, is positive-dimensional, or is infinite-dimensional. The answers to none of these questions are known at present. The author [11] has recently shown that the space of homeomorphisms of the pseudo-arc contains no nondegenerate compact connected subsets. For comparison [4], the space of homeomorphisms $H(M)$ of the Menger universal curve is totally disconnected, and one can further show that if $f$ and $g$ are distinct elements of $H(M)$ then there is a separation $H(M)=A \cup B$ with $f \in A, g \in B$, and $\operatorname{dist}(A, B)>0$.

While we do not know whether the space of homeomorphisms $H(P)$ of the pseudo-arc is totally disconnected, though we suspect that it is, we do know that $H(P)$ does not have any separation as described above for any pair of distinct elements. This is contained in the following result.

Theorem 2.1. If $\epsilon>0$ and $h: P \rightarrow P$ is a homeomorphism of the pseudo-arc $P$, then $h$ is a finite product of $\epsilon$-homeomorphisms.

Proof. Let $C$ be a chain covering the pseudo-arc of mesh less than $\epsilon / 2$. If $f$ and $g$ are homeomorphisms of $P$ such that $f(x)$ and $g(x)$ are in intersecting elements of $C$ for each $x \in P$, then $g f^{-1}$ is an $\epsilon$-homeomorphism of $P$. This observation will be used to gradually move from the identity to $h$ by a sequence of $\epsilon$-homeomorphisms.

Let $x$ be a fixed point of $h$. Let $D$ be a chain covering $P$ such that both $D$ and $h(D)$ closure refine $C$, and $D$ consists of two subchains $D_{A}$ and $D_{B}$ satisfying the following conditions:

(1) $D_{A}$ and $D_{B}$ have only one link, $D_{x}$, in common, with $x \in D_{x}$, and

(2) there are patterns $f$ and $g$ which $D$ and $h(D)$ respectively follow in $C$ such that $f$ and $g$ agree on $D_{x}, f\left(D_{A}\right)=C$, and $g\left(h\left(D_{B}\right)\right)=C$. 
Such a chain $D$ can be obtained as follows. Let $E$ be a chain covering $P$ such that both $E$ and $h(E)$ closure refine $C$ and are of sufficiently small mesh that if $E_{x}$ is a link of $E$ containing $x$ then $E_{x}$ and $h\left(E_{x}\right)$ are contained in a common link of $C$. Let $F$ be a chain covering $P$ and closure refining $E$ such that if $F_{x}$ is a link of $F$ containing $x$, then $F_{x}$ separates $F$ into two subchains each of which runs essentially through every link of $E$. The desired chain $D$ has the same properties as $F$.

There is a sequence of patterns $f=f_{0}, f_{1}, f_{2}, \ldots, f_{n}=g$ such that for each $i<n, f_{i}$ and $f_{i+1}$ disagree on only one link of $D$ and for each link $D(j)$ of $D$, $f_{i}(D(j)) \cap f_{i+1}(D(j)) \neq 0$. (This sequence can be formed by first keeping $D_{A}$ fixed and gradually changing $\left.f\right|_{D_{B}}$ to $\left.g\right|_{D_{B}}$-first retracting $\left.f\right|_{D_{B}}$ along its path back to $f\left(D_{x}\right)$ then expanding out along the path of $\left.g\right|_{D_{B}}$. Then $\left.g\right|_{D_{B}}$ is kept fixed while one similarly changes $\left.f\right|_{D_{A}}$ to $\left.g\right|_{D_{A}}$.)

By Lemma 2 there is for each $i$ a chain $E_{i}$ covering $P$ and following the pattern $f_{i}$ in $C$. Let $C_{2}$ be a chain covering $P$ crooked in $D$ such that for some pattern $g, g\left(\operatorname{st}\left(C_{2}(n), C_{2}\right)\right) \subset D(g(n))$ for each $n$. Let $D_{2}$ be a similar chain following the same pattern in $E$. By letting $D, E_{i}, C_{2}, D_{2}$, and $g$ play the role of $C_{1}, D_{1}, C_{2}, D_{2}$, and $f_{1}$ respectively in Lemma 1.1 , one can for each $i \leq n$ construct a homeomorphism $h_{i}: P \rightarrow P$ such that $h_{i}(D)$ follows the pattern $f_{i}$ in $C$. This can be done such that $h_{0}=$ identity and $h_{n}=h$. By the initial observation, $h_{i+1} h_{i}^{-1}$ is an $\epsilon$ homeomorphism for each $i \leq n$, and the composition of such terms gives $h$.

3. $p$ th roots of homeomorphisms. A homeomorphism $h: P \rightarrow P$ is said to be a $p$ th root of the homeomorphism $g: P \rightarrow P$ if $h^{p}=g$. Brechner has asked if there is a nonidentity homeomorphism of the pseudo-arc with an infinite sequence of square roots, i.e. a homeomorphism $h: P \rightarrow P$ and an infinite sequence $\left\{f_{i}\right\}_{i=1}^{\infty}$ of homeomorphisms of $P$ such that $f_{1}=h \neq$ identity and $\left(f_{i+1}\right)^{2}=f_{i}$ for each $i$. We will show a slightly stronger result. This actually follows from a previous construction of the author's [10].

THEOREM 3.1. There exists a nonidentity homeomorphism $h$ of the pseudo-arc $P$ such that for any sequence $\left\{n_{i}\right\}_{i=1}^{\infty}$ of positive integers there is a sequence $\left\{f_{i}\right\}_{i=1}^{\infty}$ of homeomorphisms of $P$ such that $f_{1}=h$ and $\left(f_{i+1}\right)^{n_{i}}=f_{i}$ for each $i$.

ProOF. Let $P=\lim \left\{T_{i}, f_{i}^{i+1}\right\}$ be the pseudo-arc constructed as an inverse limit of $n$-ods, where $T_{i}$ is an $i$ !-od, and the bonding map $f_{i}^{i+1}$ consists of two stages. The arms of $T_{i}$ can be indexed by the nonnegative integers less than $i !$. The first stage is a linear map $g_{i}^{i+1}: T_{i+1} \rightarrow T_{i}$ mapping the arm of $T_{i+1}$ indexed by $m(i+1)+n$, where $0 \leq n<i+1$, onto the arm of $T_{i}$ indexed by $m$. The second stage is the crooked oscillating map $f_{i}: T_{i} \rightarrow T_{i}$ described in [10].

As observed in [10] this construction induces natural homeomorphisms $h_{i}: P \rightarrow$ $P$ of period $i$ ! for each $i$. It is easily checked that $\left(h_{i+1}\right)^{i+1}=h_{i}$ for each $i$. Let $h_{2}$ be the desired $h$, and use appropriate powers of later $h_{j}$ 's for the $f_{i}$ 's.

A similar argument can be made for any of the other periodic homeomorphisms of the pseudo-arc constructed earlier by the author. The author does not know whether this is also true of those recently constructed by Juan Toledo [13].

4. Lifting maps of chainable continua. Here we show that every map between chainable continua can be lifted to a homeomorphism of the pseudo-arc. This result was suggested to me by questions raised in discussions with Brechner and Toledo. There are two cases to consider: (1) where the domain and range are the 
same continuum (or the range is a subcontinuum of the domain), and (2) where the domain and range are distinct continua. The first case is the more interesting, and so we consider it first.

THEOREM 4.1. If $f: X \rightarrow X$ is a map of the chainable continuum $X$ into itself, then there exists a homeomorphism $h: P \rightarrow P$ of the pseudo-arc into itself and $a$ continuous surjection $\phi: P \rightarrow X$ such that $f \phi=\phi h$.

Proof. There are sequences of chains $\left\{A_{i}\right\}_{i=1}^{\infty},\left\{B_{i}\right\}_{i=1}^{\infty},\left\{C_{i}\right\}_{i=1}^{\infty}$, and $\left\{\hat{C}_{i}\right\}_{i=1}^{\infty}$, in part determined by the map $f: \underline{\bar{X}} \rightarrow \underline{\bar{X}}$, such that:

(1) each $A_{i}$ and $C_{i}$ covers $\underline{\bar{X}}$, each $B_{i}$ irreducibly covers $f(\underline{\bar{X}})$ and consists of links which are open in $\underline{\bar{X}}$, not just in $F(\underline{\bar{X}})$, and each $\hat{C}_{i}$ is a subchain of $C_{i}$ irreducibly covering $f(\underline{\bar{X}})$,

(2) $A_{i}$ and $B_{i}$ have the same number of links,

(3) $\operatorname{mesh}\left(B_{i}\right)<1 / i$ and $\operatorname{mesh}\left(C_{i}\right)<1 / i$ for each $i$,

(4) $C_{i}$ closure refines $A_{i}$ for each $i$,

(5) $B_{i+1}$ closure refines $\hat{C}_{i}$ for each $i$,

(6) $\hat{C}_{i+1}$ closure refines $B_{i}$ for each $i$,

(7) for each $i$ there exist patterns $\alpha_{i}, \beta_{i}, \gamma_{i}, \delta_{i}$, and $\epsilon_{i}$ such that $A_{i+1}$ follows the pattern $\alpha_{i}$ in $A_{i}, B_{i+1}$ follows the pattern $\alpha_{i}$ in $B_{i}, C_{i}$ follows the pattern $\beta_{i}$ in $A_{i}, C_{i+1}$ follows the pattern $\gamma_{i}$ in $C_{i}, B_{i+1}$ follows the pattern $\delta_{i}$ in $\hat{C}_{i}$, and $\hat{C}_{i+1}$ follows the pattern $\epsilon_{i}$ in $B_{i}$, and

(8) $f\left(A_{i}(n)\right)=B_{i}(n) \cap f(\underline{\bar{X}})$ for each link $A_{i}(n)$ of $A_{i}$. (Recall that the links of $B_{i}$ are enlarged slightly to be open in $\underline{\bar{X}}$, not just in $f(\underline{\bar{X}})$.)

By repeated application of Lemmas 1 and 2 from the introduction, there exist a pseudo-arc $P$ and sequences of chains $\left\{D_{i}\right\}_{i=1}^{\infty},\left\{E_{i}\right\}_{i=1}^{\infty},\left\{F_{i}\right\}_{i=1}^{\infty},\left\{\hat{F}_{i}\right\}_{i=1}^{\infty},\left\{G_{i}\right\}_{i=1}^{\infty}$ and $\left\{H_{i}\right\}_{i=1}^{\infty}$ satisfying each of the conditions listed below. Chains $D_{i}, E_{i}, F_{i}$, and $\hat{F}_{i}$ will be the lifted versions of $A_{i}, B_{i}, C_{i}$ and $\hat{C}_{i}$ respectively. Since each of these latter chains can be viewed as an amalgamation of an appropriate $C_{j}$ it is enough to know by Lemma 2 that we can lift $C_{j}$ to form the chain $F_{j}$ following the appropriate pattern. The chains $G_{i}$ and $H_{i}$ are introduced to be able to construct the homeomorphism of the pseudo-arc. The introductory lemmas and techniques similar to those already used guarantee that we can construct such chains satisfying the desired conditions.

These are the conditions to be satisfied.

(1) Each $D_{i}, F_{i}$, and $G_{i}$ covers $P$, while there is a fixed subcontinuum $\hat{P}$ of $P$ that each $E_{i}, \hat{F}_{i}$, and $H_{i}$ irreducibly covers. $\hat{F}_{i}$ is a subchain of $F_{i}$ which irreducibly covers $\hat{P}$.

(2) $D_{i}, E_{i}, F_{i}$, and $\hat{F}_{i}$ have the same number of links as $A_{i}, B_{i}, C_{i}$, and $\hat{C}_{i}$ respectively.

(3) $F_{i}(n)$ is a link of $\hat{F}_{i}$ if and only if $C_{i}(n)$ is a link of $\hat{C}_{i}$.

(4) For each $i, G_{i}$ and $H_{i}$ have the same number of links.

(5) For each $i, \operatorname{mesh}\left(G_{i}\right)<1 / i$ and $\operatorname{mesh}\left(H_{i}\right)<1 / i$. Though $B_{i}$ and $C_{i}$ have small mesh, their lifted versions, $E_{i}$ and $F_{i}$, need not have small mesh.

(6) For each $i, F_{i}$ closure refines $D_{i}$.

(7) For each $i, E_{i+1}$ closure refines $\hat{F}_{i}$.

(8) For each $i, \hat{F}_{i+1}$ closure refines $E_{i}$.

(9) For each $i, G_{i}$ closure refines $F_{i}$. 
(10) For each $i, H_{i}$ closure refines $E_{i}$.

(11) For each $i, D_{i+1}$ follows the pattern $\alpha_{i}$ in $D_{i}, E_{i+1}$ follows the pattern $\alpha_{i}$ in $E_{i}, F_{i}$ follows the pattern $\beta_{i}$ in $D_{i}, F_{i+1}$ follows the pattern $\gamma_{i}$ in $F_{i}, E_{i+1}$ follows the pattern $\delta_{i}$ in $\hat{F}_{i}$, and $\hat{F}_{i+1}$ follows the pattern $\epsilon_{i}$ in $E_{i}$.

(12) For each $i$, there exist patterns $\zeta_{i}$ and $\eta_{i}$ such that $G_{i+1}$ follows the pattern $\zeta_{i}$ in $G_{i}, H_{i+1}$ follows the pattern $\zeta_{i}$ in $H_{i}, G_{i}$ follows the pattern $\eta_{i}$ in $F_{i}$, and $H_{i}$ follows the pattern $\beta_{i} \eta_{i}$ in $E_{i}$.

By Lemma 3 of the introduction and the remarks after it, the sequence of patterns $\left\{\zeta_{i}\right\}_{i=1}^{\infty}$ and the sequences of chains $\left\{G_{i}\right\}_{i=1}^{\infty}$ and $\left\{H_{i}\right\}_{i=1}^{\infty}$ determine a homeomorphism $h: P \rightarrow \hat{P}$. The sequences of chains $\left\{C_{i}\right\}_{i=1}^{\infty}$ and $\left\{G_{i}\right\}_{i=1}^{\infty}$ and the sequence of patterns $\left\{\gamma_{i}\right\}_{i=1}^{\infty}$ determine a continuous surjection $\phi: P \rightarrow \underline{X}$.

The sequences $\left\{B_{i}\right\}_{i=1}^{\infty},\left\{E_{i}\right\}_{i=1}^{\infty}$, and $\left\{\alpha_{i}\right\}_{i=1}^{\infty}$ determine a continuous surjection $\hat{\phi}: \hat{P} \rightarrow f(\underline{\bar{X}})$. With the sequences $\left\{\hat{C}_{i}\right\}_{i=1}^{\infty},\left\{\hat{F}_{i}\right\}_{i=1}^{\infty},\left\{G_{i}\right\}_{i=1}^{\infty},\left\{H_{i}\right\}_{i=1}^{\infty},\left\{\delta_{i}\right\}_{i=1}^{\infty}$, $\left\{\epsilon_{i}\right\}_{i=1}^{\infty},\left\{\zeta_{i}\right\}_{i=1}^{\infty}$, and $\left\{\eta_{i}\right\}_{i=1}^{\infty}$, they guarantee that $\left.\phi\right|_{\hat{P}}=\hat{\phi}$ and that $f \phi=\hat{\phi} h=\phi h$.

If, as in the above theorem, $f(\underline{X})=\underline{\bar{X}}$, then the construction can be done such that $\hat{P}=P$. If $f: \underline{X} \rightarrow Y$ is a map of chainable continua where $Y$ is not a subcontinuum of $\underline{\bar{X}}$, then one can get a similar but not as strong (and much easier) result, which we state without proof.

THEOREM 4.2. If $X$ and $Y$ are chainable continua and $f: X \rightarrow Y$ is a map, then there exists a pseudo-arc $P$, continuous surjections $\phi: P \rightarrow X$ and $\psi: P \rightarrow f(X)$, and a homeomorphism $h: P \rightarrow P$ such that $f \phi=\psi h$.

In general, if $X$ is a chainable continuum and $P$ a pseudo-arc, there are many continuous surjections $\phi: P \rightarrow X$. In the two previous theorems the surjections $\phi$ and $\psi$ were determined by the map $f$, and were not given beforehand. It is in general not true that if $\bar{\phi}: P \rightarrow X$ is a continuous surjection of the pseudo-arc $P$ onto the chainable continuum $X$ and $f: X \rightarrow X$ is a map of $X$ into itself, then there exists a homeomorphism (or even map) $h: P \rightarrow P$ with $f \bar{\phi}=\bar{\phi} h$. For example, one can easily construct a projection $\bar{\phi}: P \rightarrow I$ of the pseudo-arc onto the unit interval with the preimages of the opposite endpoints of $I$ nonhomeomorphic.

For any such maps $\bar{\phi}$ and $f$ and any $\epsilon>0$, one can construct a homeomorphism $h: P \rightarrow P$ giving $\epsilon$-commutativity, i.e., $\operatorname{dist}(f \bar{\phi}, \bar{\phi} h)<\epsilon$. Every map onto a chainable continuum is weakly confluent, and so there is a subcontinuum $\hat{P}$ of $P$ such that $\bar{\phi}(\hat{P})=f(\underline{X})=f \bar{\phi}(P)$. If $C$ is a chain of mesh less than $\epsilon$ which covers $f(\underline{X})$, then $(f \bar{\phi})^{-1}(C)$ and $\bar{\phi}^{-1}(C) \cap \hat{P}$ are chains, with the same number of links, covering $P$ and $\hat{P}$ respectively. If $\hat{P}$ is nondegenerate, then techniques similar to Lemma 3 or Lemma 1.1 give the desired homeomorphism $h$. If $\hat{P}$ is a single point, let $h$ be a homeomorphism of $P$ onto a subcontinuum containing $\hat{P}$ which is sufficiently small so that $\bar{\phi} h(P)$ has diameter less than $\epsilon$.

5. Questions. The results in the previous four sections raise several questions which we pose here.

The collection of continua which are pseudo-arcs and the collection of maps of the pseudo-arc which are homeomorphisms are large in the sense of category. Are they also large in the sense of measure?

Question 1. Is there a natural measure which can be put on the space $M(P)$ of maps of the pseudo-arc? If so, what is the measure of the subspace $\hat{H}(P)$ ? Is it the same as the measure of $M(P)$ ? 
Question 2. If $E$ is a Euclidean space or Hilbert space of dimension greater than 1 , is there a natural measure on the hyperspace $C(E)$ of subcontinua of $E$ ? If so, what is the measure of the subspace of $C(E)$ consisting of all pseudo-arcs in $E$ ? Is it the same as the measure of $C(E)$ ?

Similar questions can be asked about $M C(E)$ where $\operatorname{dim}(E) \geq 3$.

Question 3. Which other nondegenerate continua $X$ have the property that $\hat{H}(X)$ is a dense $G_{\delta}$ in $M(X)$ ?

We still know very little about the structure of the space of homeomorphisms $H(P)$ of the pseudo-arc $P$.

Question 4. Is $H(P)$ totally disconnected? If $f$ and $g$ are distinct elements of $H(P)$ is there a separation $H(P)=A \cup B$ with $f \in A$ and $g \in B$ ? Is $H(P)$ infinite dimensional?

It is easy to see that $M(P)$ is not totally disconnected, and in fact contains nondegenerate subcontinua (e.g. the set of all constant maps from $P$ into $P$ is itself homeomorphic to $P$ ). Still, more information about its structure, and how $H(P)$ and $\hat{H}(P)$ sit as subspaces of it, would be of interest.

Question 5. Does there exist a connected subset of $M(P)$ containing distinct elements of $H(P)$ ? of $\hat{H}(P)$ ?

Question 6. Does every periodic homeomorphism $h$ of the pseudo-arc have an $n$ th-root for each positive integer $n$ ? If $n>1$ and $k^{n}=h$, where $h$ has a nondegenerate fixed point set, can be the fixed point set of $k$ always be made smaller than that of $h$ ?

A continuum is weakly chainable $[\mathbf{7}, \mathbf{8}]$ if it is the continuous image of a chainable continuum, in particular the image of a pseudo-arc.

Question 7. If $X$ is a weakly chainable, tree-like continuum and $f: X \rightarrow X$ is continuous, does there exist a continuous surjection $\phi: P \rightarrow X$ of the pseudo-arc onto $X$ and a homeomorphism (or map) (not necessarily surjective) $h: P \rightarrow P$ such that $f \phi=\phi h$ ?

A positive answer to Question 7 would show that weakly chainable, tree-like continua have the fixed point property, but this might be the difficult way to try to show such a result. For Question 7, it may prove convenient to look at the pseudo-arc with a defining sequence of tree chains rather than simple chains.

Question 8. Which of the results presented here extend to continua of span zero (in case there are any such which are not chainable)?

\section{REFERENCES}

1. R. H. Bing, A homogeneous indecomposable plane continuum, Duke Math. J. 15 (1948), 729-742.

2. __ Concerning hereditarily indecomposable continua, Pacific J. Math. 1 (1951), 43-51.

3. __ Snake-like continua, Duke Math. J. 18 (1951), 653-663.

4. B. Brechner, On the dimensions of certain spaces of homeomorphisms, Trans. Amer. Math. Soc. 121 (1966), 516-548.

5. __ Homeomorphism groups of chainable and homogeneous continua, Topology Proc. 8 (1983).

6. H. Cook, Continua which admit only the identity mapping onto nondegenerate subcontinua, Fund. Math. 60 (1967), 241-249.

7. L. Fearnley, Characterizations of the continuous images of the pseudo-arc, Trans. Amer. Math. Soc. 111 (1964), 380-399.

8. A. Lelek, On weakly chainable continua, Fund. Math. 51 (1962), 271-282.

9. W. Lewis, Stable homeomorphisms of the pseudo-arc, Canad. J. Math. 31 (1979), 363-374.

10. __ Periodic homeomorphisms of chainable continua, Fund. Math. 117 (1982). 
11. __ Pseudo-arcs and connectedness in homeomorphism groups, Proc. Amer. Math. Soc. 87 (1983), 745-748.

12. E. E. Moise, An indecomposable plane continuum which is homeomorphic to each of its nondegenerate subcontinua, Trans. Amer. Math. Soc. 63 (1948), 581-594.

13. J. Toledo, Finite and compact actions on chainable and tree-like continua, Ph.D. Dissertation, Univ. of Florida, 1982.

Department of Mathematics, Texas Tech University, Lubbock, Texas 79409 